\title{
Silicon Nanocrystal Synthesis in Microplasma Reactor*
}

\author{
Tomohiro NOZAKI**, Kenji SASAKI**, Tomohisa OGINO**, Daisuke ASAHI** \\ and Ken OKAZAKI** \\ **Department of Mechanical and Control Engineering, Tokyo Institute of Technology \\ 2-12-1 O-okayama, Meguro, Tokyo Japan, 152-8552 \\ E-mail: tnozaki@mech.titech.ac.jp
}

\begin{abstract}
Nanocrystalline silicon particles with grains smaller than $5 \mathrm{~nm}$ are widely recognized as a key material in optoelectronic devices, lithium battery electrodes, and bio-medical labels. Another important characteristic is that silicon is an environmentally safe material that is used in numerous silicon technologies. To date, several synthesis methods such as sputtering, laser ablation, and plasma-enhanced chemical vapor deposition (PECVD) based on low-pressure silane chemistry $\left(\mathrm{SiH}_{4}\right)$ have been developed for precise control of size and density distributions of silicon nanocrystals. In this study, we explore the possibility of microplasma technologies for efficient production of mono-dispersed nanocrystalline silicon particles on a micrometer-scale, continuous-flow plasma reactor operated at atmospheric pressure. Mixtures of argon, hydrogen, and silicon tetrachloride were activated using a very-high-frequency $(144 \mathrm{MHz})$ power source in a capillary glass tube with volume of less than $1 \mu \mathrm{l}$. Fundamental plasma parameters of the microplasma were characterized using optical emission spectroscopy, which respectively indicated electron density of $10^{15} \mathrm{~cm}^{-3}$, argon excitation temperature of $5000 \mathrm{~K}$, and rotational temperature of $1500 \mathrm{~K}$. Such high-density non-thermal reactive plasma can decompose silicon tetrachloride into atomic silicon to produce supersaturated silicon vapor, followed by gas-phase nucleation via three-body collision: particle synthesis in high-density plasma media is beneficial for promoting nucleation processes. In addition, further growth of silicon nuclei can be terminated in a short-residence-time reactor. Micro-Raman scattering spectra showed that as-deposited particles are mostly amorphous silicon with a small fraction of silicon nanocrystals. Transmission electron micrography confirmed individual 3-15 nm silicon nanocrystals. Although particles were not mono-dispersed, they were well separated and not coagulated.
\end{abstract}

Key words: Microplasma, Atmospheric Pressure Non-Equilibrium Plasma, Silicon Nanocrystals

\section{Introduction}

A microplasma is characterized as a high-density non-thermal plasma generated in a miniaturized reactor whose characteristic length is generally less than one millimeter ${ }^{(1)}$. Unlike conventional low-pressure plasma sources, microplasma requires a high-density medium such as atmospheric pressure gas, liquid, or supercritical fluid so that electrons and molecules have sufficient interactions to initiate electrical breakdown. The use of microplasma currently attracts broad attention from industry. Fundamental research and a range of applications including waste gas treatment, micro-fabrication in open air, and liquid-phase bio-medical treatment are being investigated. We explore the possibility of microplasma technologies for efficient production of mono-dispersed nanocrystalline silicon

${ }^{*}$ Received 12 June, 2007 (No. T1-TSE-1443) Japanese Original : Thermal Sci. \& Eng., Vol.14, No.4 (2006),

pp.75-81 (Received 9 Mar., 2006) [DOI: 10.1299/jtst.2.192] 
particles in a continuous flow microplasma reactor operated at atmospheric pressure ${ }^{(2)}$.

Silicon nanocrystals with grains smaller than $5 \mathrm{~nm}$ are widely recognized as a key material in optoelectronics, bio-imaging, and photovoltaic applications. In general, silicon cannot emit visible light because it is an indirect transition semiconductor. However, it can emit visible light because of the quantum size effect if its crystal become smaller than $5 \mathrm{~nm}$. The band gap of a 2-nm fully hydrogenated silicon nanocrystal is estimated as $2.4 \mathrm{eV}$; visible light centered around $517 \mathrm{~nm}$ can be elicited when silicon nanocrystals are stimulated by ultraviolet irradiation. To date, several synthesis methods such as sputtering, laser ablation, and plasma-enhanced chemical vapor deposition based on low-pressure processing ${ }^{(3-6)}$ have been established for synthesis of silicon nanostructured materials. Colloidal growth using organosilane is another possibility; however, silicon nanocrystals are obtained only in small amounts because silicon carbides and silicon oxides are preferentially synthesized. We propose a microplasma approach as a viable synthesis technique of tunable photoluminescent silicon nanocrystals. In high-density reactive plasma media, the silicon precursor can be fully decomposed to create supersaturated silicon vapor, followed by rapid crystal nucleation via three-body collision. Furthermore, excessive growth of silicon nuclei is avoidable in a short-residence-time reactor. In the first section of this paper, the experimental setup of a microplasma reactor with volume of less than $1 \mu \mathrm{l}$ will be presented. Optical emission spectroscopy was applied for estimation of plasma parameters such as electron density, rotational temperature, and the excitation temperature of argon. The effect of residence time on nanocrystal synthesis is discussed in detail. Finally, concluding remarks are presented.

\section{Paschen's law - similarity rule}

Figure 1 shows the gas breakdown voltage with respect to the $(p d)$ product known as the Paschen curve ${ }^{(7)}$. In that figure, $d$ represents the electrode separation in centimeters and $p$ represents total pressure in millimeters of mercury. The Paschen curve in DC breakdown is determined by the cathode material and molecule type; each curve should take a minimal value of $p d=1-10(\mathrm{mmHg} \mathrm{cm})$. The Paschen curve is also know as a similarity rule. Such a relation can be validated empirically in a wide range of conditions ${ }^{(8)}: p=10^{-2}$ Torr -2400 Torr; $d=5 \times 10^{-4} \mathrm{~cm}-20 \mathrm{~cm}$; gas temperature $=-15^{\circ} \mathrm{C}-860^{\circ} \mathrm{C}$. Gap separation and the electron mean free pass come to be of a comparable order when the $(p d)$ product is smaller than $(p d)_{\min }$. Therefore, electrons are unable to receive sufficient energy from electric field to ionize gaseous molecules. As a result, the breakdown voltage is sharply elevated as the ( $p d$ ) value decreases. In contrast, when $(p d)>(p d)_{\min }$, electrons lose their energy via excessive collisions with ambient molecules, engendering a rather moderate increase in the breakdown voltage as the $(p d)$ value increases. Table 1 lists the breakdown voltages, $(p d)_{\min }$, and $d_{\min }$ at $760 \mathrm{mmHg}$ for various gases ${ }^{(7)}$. The minimum gap separation, $d_{m i n}$, at $760 \mathrm{mmHg}$ for

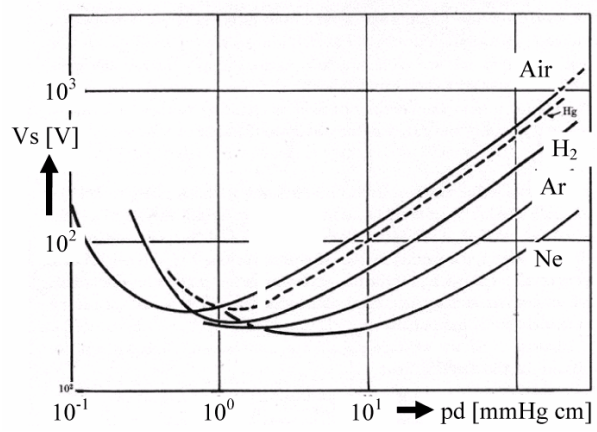

Fig. 1 Breakdown voltage vs. Reduced electrode distance $(p d)$ : Paschen curve ${ }^{(7)}$. monatomic molecules is approximately $30 \mu \mathrm{m}$; that for polyatomic molecules is $10 \mu \mathrm{m}$. Consequently, if the gap separation becomes smaller than $d_{\text {min }}$ (= 10-30 $\mu \mathrm{m})$, the operating pressure would have to be much greater than 760 Torr to initiate electrical breakdown. Paschen's law is extended by introducing the $(f d)$ parameter, where $f$ represents the operating frequency $[\mathrm{Hz}]$ ${ }^{(9)}$. The $(p d)_{\min }$ value decreases with frequency because trapped ions and electrons in the gap contribute to reduce 
the breakdown voltage in the given conditions. Unlike $(p d)$ dependence, the breakdown voltage does not have a minimum value with respect to $(f d)$. For this study, the operating pressure was fixed at atmospheric pressure $(760 \mathrm{mmHg})$; the reactor dimensions were various, from $290 \mu \mathrm{m}$ to $800 \mu \mathrm{m}$, which is slightly larger than $(p d)_{\min }$ given at 760 Torr.

Table 1 Minimum breakdown potential and electrode distance at 760 Torr $^{(7)}$.

\begin{tabular}{ccccc}
\hline $\begin{array}{c}\text { Gas } \\
\text { medium }\end{array}$ & $\begin{array}{c}\text { Cathode } \\
\text { material }\end{array}$ & $\begin{array}{c}V_{\min } \\
(\mathrm{V})\end{array}$ & $\begin{array}{c}\mathrm{pd} \\
(\text { Torr-cm })\end{array}$ & $\begin{array}{c}d_{\min } \\
(\mu \mathrm{m})\end{array}$ \\
\hline $\mathrm{He}$ & $\mathrm{Fe}$ & 150 & 2.5 & 32.9 \\
$\mathrm{Ne}$ & $"$ & 244 & 3 & 39.5 \\
$\mathrm{Ar}$ & $"$ & 265 & 1.5 & 19.7 \\
\hline $\mathrm{N}_{2}$ & $"$ & 275 & 0.75 & 9.9 \\
$\mathrm{O}_{2}$ & $"$ & 450 & 0.7 & 9.2 \\
$\mathrm{Air}$ & $"$ & 330 & 0.57 & 7.5 \\
$\mathrm{H}_{2}$ & $\mathrm{Pt}$ & 295 & 1.25 & 16.4 \\
$\mathrm{CO}_{2}$ & $?$ & 420 & 0.5 & 6.6 \\
$\mathrm{Hg}$ & $\mathrm{W}$ & 425 & 0.8 & 10.5 \\
$\mathrm{Hg}$ & $\mathrm{Fe}$ & 520 & 2 & 26.3 \\
$\mathrm{Na}$ & $\mathrm{Fe} ?$ & 335 & 0.04 & 0.53 \\
\hline
\end{tabular}

?: Unknown

\section{Experimental}

Figure 2 and 3 respectively show schematic diagrams of the experimental setup and a close-up image of microplasma. A pair of electrodes was installed outside of the capillary glass tube (O.D. $1100 \mu \mathrm{m}$, I.D. $290-800 \mu \mathrm{m}$ ) to avoid metallic contamination of the reaction zone. A miniaturized, capacitively-coupled non-thermal discharge was generated inside the capillary tube using a very-high-frequency (VHF: $144 \mathrm{MHz}$ ) power source equipped with $\pi$-type matching network. A VHF operation enables trapping of charges in the gap and creates quasi-continuous plasma conditions. Microplasma is not confined in the flow direction; therefore, the emission region extends freely beyond the metallic electrodes, as shown in Fig. 3. In this respect, the distance between electrodes is not as important as the capillary tube diameter. We also found that the electron density and excitation temperature were almost independent with respect to gap separation; for that reason, it was fixed at $4 \mathrm{~mm}$ throughout the experiments.

A mixture of argon, silicon tetrachloride, and hydrogen was fed into the microplasma reactor via the mixing chamber. Hydrogen efficiently promotes chlorine abstraction from

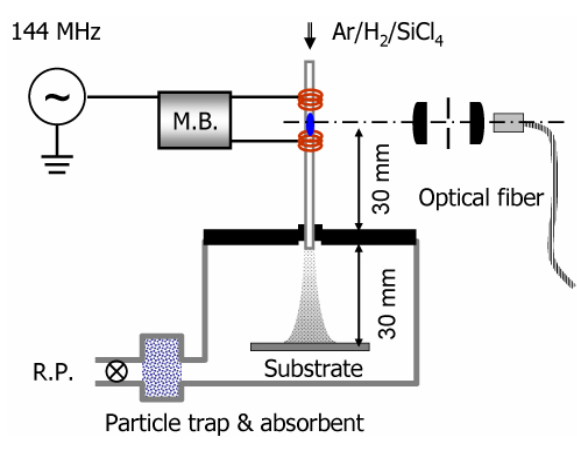

Fig. 2 Schematic diagram of microplasma reactor.

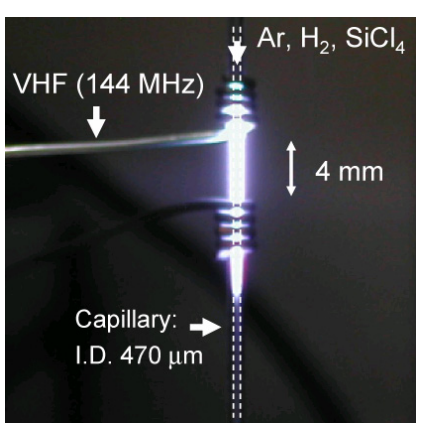

Fig. 3 A close-up image of microplasma: Ar, $1000 \mathrm{~cm}^{3} \mathrm{~min}^{-1} ; \mathrm{H}_{2}$, $10 \mathrm{~cm}^{3} \mathrm{~min}^{-1}$; power, $15 \mathrm{~W}$. 
$\mathrm{SiCl}_{\mathrm{n}}$ and contributes to creation of supersaturated silicon vapor. Furthermore, hydrogen terminates the unreacted silicon bond of the cluster surface and produces smaller silicon nanocrystals. Silicon nanocrystals formed in the gas phase were deposited on various substrates depending on the material characterization technique. As described later, the gas temperature of the microplasma was elevated to $1500 \mathrm{~K}$. Therefore, the distance between the microplasma and the substrate $(60 \mathrm{~mm})$ must be great so that the heated working gas does not induce secondary nucleation in the as-synthesized material. Silicon nanocrystals were characterized using a scanning electron microscope (S-800; Hitachi Ltd.) and a transmission electron microscope (200 kV, JEM-3010; JEOL). Optical emission spectroscopy was used for estimation of microplasma properties. Emission from microplasma was introduced into a spectrometer (Spectrapro-2750 equipped with 1200 line $\mathrm{mm}^{-1}$ grating; Acton Research Corp.); data were recorded using a CCD camera (iDus DV420A-BU; Andor Technology).

\section{Optical emission spectroscopy of microplasma}

Fundamental plasma parameters such as gas temperature and electron density were estimated using optical emission spectroscopy (OES). In the OES analysis, spontaneous emissions from individual known species were analyzed. The relative intensity ratios of several emission lines or line width provide semiquantitative information about plasma parameters. Detailed numerical modeling or alternative diagnostics are necessary, such as that by absorption spectroscopy, for quantitative evaluation of given data. Nevertheless, OES is widely recognized as a powerful tool for plasma characterization because it allows in situ measurements using simple optical settings ${ }^{(10,11)}$.

\subsection{Gas temperature}

A trace amount of methane was added for analysis of the rotational temperature of $\mathrm{CH}$ $(432 \mathrm{~nm})$; it reasonably reflects the gas temperature of the corresponding emission region $(12,13)$. The rotational temperature was determined using the Boltzmann plot method, where the relative intensity ratio of the individual rotational line is plotted against the upper rotational energy. If excited $\mathrm{CH}$ establish local thermal equilibrium with respect to translational temperature of surrounding molecules via tens of collisions, the Boltzmann plot provides a fairly straight line and rotational temperatures are estimated from the inverse of the slope. Methane was not injected into the microplasma during silicon nanoparticle synthesis.

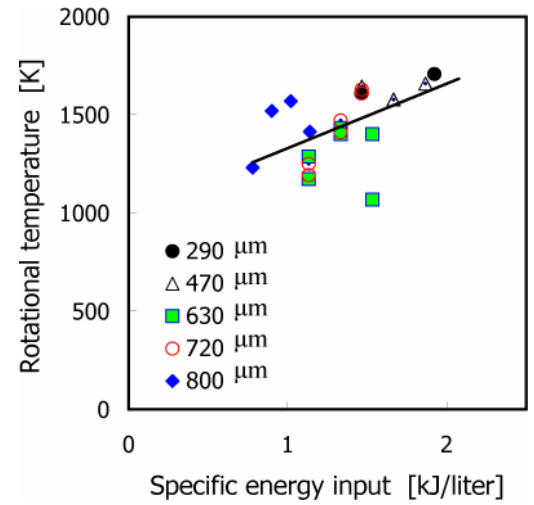

Fig. 4 Rotational temperature of $\mathrm{CH}$ with respect to specific energy input. Ar, 1000 $\mathrm{cm}^{3} \min ^{-1} ; \mathrm{CH}_{4}, 1 \mathrm{~cm}^{3} \min ^{-1} ; \mathrm{H}_{2}, 5 \mathrm{~cm}^{3}$ $\min ^{-1}$; power, $15-25 \mathrm{~W}$.

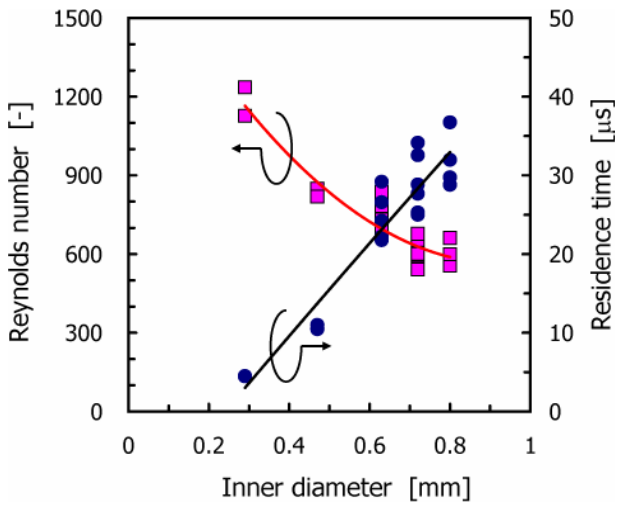

Fig. 5 Reynolds number and residence time with respect to capillary inner diameter. Conditions: see Fig. 4 
Results obtained using different capillary diameters are shown in Fig. 4. The rotational temperature increases from $1200 \mathrm{~K}$ to $1700 \mathrm{~K}$ as the input energy per flow rate increases. Assuming plug flow, the average gas flow rate was estimated using the following equation:

$$
u_{\text {ave }}=\frac{Q}{60 \times A_{c}}\left(\frac{T_{r o t}}{273}\right) \text {. }
$$

In that equation, $Q$ is the total gas flow rate $\left[\mathrm{cm}^{3} \mathrm{~min}^{-1}\right], A_{c}$ is the cross-section area of the capillary glass $\left[\mathrm{cm}^{2}\right]$, and $T_{r o t}$ is the rotational temperature of $\mathrm{CH}[\mathrm{K}]$. The residence time and Reynolds number were estimated using the physical properties of argon at a given rotational temperature (Fig. 5).

$$
\operatorname{Re}=\frac{u_{a v e} \times d_{c}}{v}=\frac{1}{15 \pi} \times\left(\frac{Q}{v d_{c}}\right) \times\left(\frac{T_{r o t}}{273}\right)
$$

Therein, $d_{c}$ is the internal diameter of the capillary glass [cm] and $v$ is the kinematic viscosity coefficient of argon estimated at a given rotation temperature $\left[\mathrm{cm}^{2} \mathrm{~s}^{-1}\right]$. The Reynolds number increases monotonically up to 1200 with decreasing capillary diameter. Although detailed flow patterns under the influence of non-thermal discharge are not fully understood, we assumed a laminar flow because we did not observe abrupt changes in plasma properties that might be associated with lamina-to-turbulent transition. In contrast, the reaction time increases monotonically from $5 \mu$ s to $30 \mu$ s with the capillary diameter, meaning that crystal nucleation and growth were controllable in this time range. It is noteworthy that the average gas velocity approaches acoustic velocity with $290 \mu \mathrm{m}$ capillary glass. In this case, microplasma is likely to be blown off and difficult to sustain. For subsequent experiments, $470-\mu \mathrm{m}$-width capillary glass was used.

\subsection{Electron density and excitation temperature of argon}

Electron density was estimated according to the Stark broadening of $\mathrm{H}_{\beta}$ line (486 nm). The relation between line width and electron density has been well evaluated and is known to be effective for electron densities greater than $10^{14} \mathrm{~cm}^{-3}{ }^{(11)}$. The electron density of microplasma was estimated as $1-3 \times 10^{15} \mathrm{~cm}^{-3}$, which is ten times larger than $\mathrm{SiCl}_{4}$ density (ca. $10^{14} \mathrm{~cm}^{-3}$ ). The electronic excitation temperature of argon was also evaluated from the Boltzmann plot of argon emission lines ${ }^{(10,14)}$. Unlike the rotational temperature, the electronic excitation temperature is closely related to the mean electron energy. For that reason, it is frequently analyzed as a good indicator of electron temperature. However, quantitative discussion requires a detailed radiative-collision model that includes elementary excitation and deactivation steps ${ }^{(10,11)}$. The excitation temperature of argon decreased from $5000 \mathrm{~K}$ to $4000 \mathrm{~K}$ with initial hydrogen content because electrons lose their energy via various inelastic collisions with hydrogen molecules. In fact, emission from microplasma weakened gradually and was finally extinguished with excessive hydrogen contents in the main stream. Detailed data are summarized in Table 2 together with measurement conditions.

Table 2 Microplasma characteristics: capillary tube outer and inner diameters, $1100 \mu \mathrm{m}$ and $470 \mu \mathrm{m}$; reactor volume $<1 \mu \mathrm{l} ; \mathrm{Ar}, 1000 \mathrm{~cm}^{3} \mathrm{~min}^{-1} ; \mathrm{H}_{2}, 10 \mathrm{~cm}^{3} \mathrm{~min}^{-1} ; \mathrm{SiCl}_{4}, 20$ ppm; power, $15 \mathrm{~W}$.

\begin{tabular}{lll}
\hline \hline Gas temperature & ca. $1500 \mathrm{~K}$ & Boltzmann plot: CH $(432 \mathrm{~nm})$ \\
Excitation temperature of argon & $4000-5000 \mathrm{~K}$ & $\begin{array}{l}\text { Boltzmann plot: Ar I }(696.5 \mathrm{~nm}-811.5 \\
\mathrm{nm}, 415.9 \mathrm{~nm}-430 \mathrm{~nm})\end{array}$ \\
Electron density & $1-3 \times 10^{15} \mathrm{~cm}^{-3}$ & Stark broadening of $\mathrm{H}_{\beta}(486 \mathrm{~nm})$ \\
Relative concentration of $\mathrm{Si}$ & $\mathrm{Si} \approx \mathrm{SiCl}_{4}$ & Actinometry of $\mathrm{Si}(288 \mathrm{~nm})$ and Ar $(750$ \\
& $\approx 10^{14} \mathrm{~cm}^{-3}$ & $\mathrm{~nm})$ \\
Saturated concentration of $\mathrm{Si}$ & $2 \times 10^{11} \mathrm{~cm}^{-3}$ & at $1500 \mathrm{~K}$ \\
Residence time & $6 \mu \mathrm{s}$ & $100 \mathrm{kPa}, 1500 \mathrm{~K}, 1000 \mathrm{~cm}^{3} \mathrm{~min}^{-1}$ \\
\hline
\end{tabular}




\section{Silicon nanocrystal synthesis}

\subsection{Effect of reaction time}

Figure 6 presents results of elementary analyses of as-synthesized materials for argon flow rates of 200,500, and $1000 \mathrm{~cm}^{3} \mathrm{~min}^{-1}$. Figure 7 shows cross-section scanning electron micrographs (SEM) at 200 and $1000 \mathrm{~cm}^{3} \mathrm{~min}^{-1}$. The as-synthesized material was severely contaminated by chlorine and nitrogen when the argon flow rate was $200 \mathrm{~cm}^{3} \mathrm{~min}^{-1}$. Infrared absorption spectra (FT/IR-660; Jasco Inc.) shown in Fig. 8 exhibit intense absorption peaks centered around $3155 \mathrm{~cm}^{-1}$ and $1410 \mathrm{~cm}^{-1}$, which are attributed to ammonium chloride $\left(\mathrm{NH}_{4} \mathrm{Cl}\right)$. We also observed an intense emission line from $\mathrm{NH}(336 \mathrm{~nm})$ at a $200 \mathrm{~cm}^{3} \mathrm{~min}^{-1}$ argon flow rate. We speculate that nitrogen causes a residual element in the gas line because the emission band spectrum of $\mathrm{N}_{2}$, which provides a fairly strong emission line in reactive plasma, was completely missing. Once nitrogen is incorporated into the material in the form of $\mathrm{NH}_{\mathrm{i}}$, it readily reacts with $\mathrm{HCl}$ to form ammonium chloride $\left(\mathrm{NH}_{4} \mathrm{Cl}\right)$. Ammonium chloride is apparent as large agglomerates in Fig. 7(a), probably because $\mathrm{NH}_{4} \mathrm{Cl}$ absorbed moisture in air and was dissolved. Figure 7(b) shows fine powder-like deposits when the flow rate was $1000 \mathrm{~cm}^{3} \mathrm{~min}^{-1}$. Natural oxidation of the as-synthesized material was unavoidable: the sample was readily oxidized upon exposure to air; the elementary analysis shown in Fig. 6 exhibits $20-30 \%$ oxygen.

\subsection{Estimation of relative concentration of atomic silicon}

Figures 6-8 suggest that the shorter residence time favorably synthesized fine powder-like material without nitrogen and chlorine contamination. For further analysis, the relative concentration of atomic silicon was estimated using OES according to the following relationship.

$N_{S i}=C \frac{I_{S i(288 n m)}}{I_{A r(750 \mathrm{~nm})}} N_{A r}=C \times N_{S i}^{\prime}$

Here, $N$ and $I$ respectively represent the density of atoms $\left[\mathrm{cm}^{-3}\right]$ and emission intensity [-]. In addition, $N^{\prime}$ expresses the relative number density of atoms. The

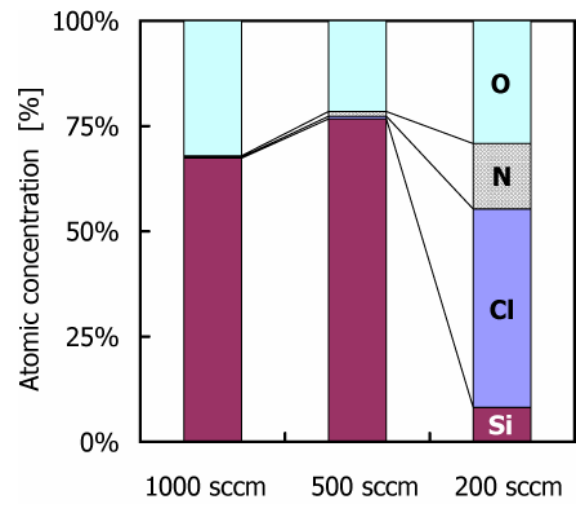

Fig. 6 Dependence of residence time on products composition: $\mathrm{SiCl}_{4}, 20 \mathrm{ppm} ; \mathrm{H}_{2}$, 10000 ppm; power, 10-20 W.
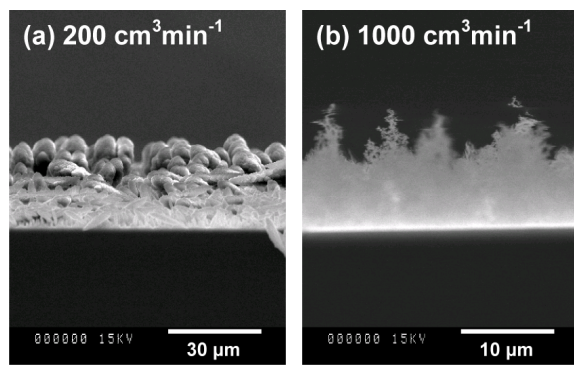

Fig. 7 Cross-section view of as-synthesized products. $\mathrm{SiCl}_{4}, 20 \mathrm{ppm}$; $\mathrm{H}_{2}, 10000$ ppm; power, 10-20 W.

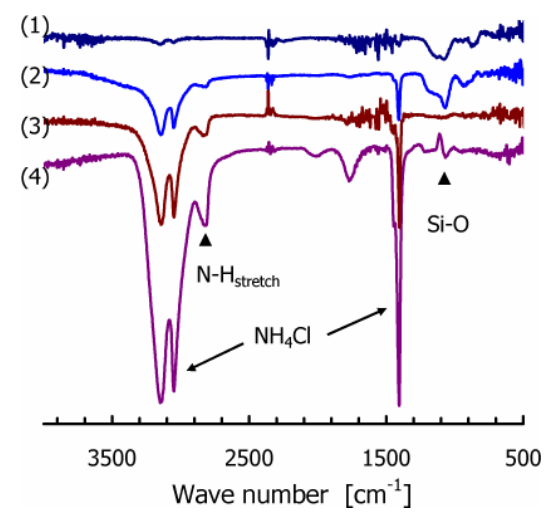

Fig. 8 FTIR Spectra of as-synthesized samples with $\mathrm{SiCl}_{4} 20$ ppm: (1) Ar, 1000 sccm - $\mathrm{H}_{2}, 10^{4}$ ppm; (2) Ar, $500 \mathrm{sccm}$ $\mathrm{H}_{2}, 10^{4} \mathrm{ppm}$; (3) Ar, $200 \mathrm{sccm}-\mathrm{H}_{2}, 2 \times 10^{4}$ ppm; (4) Ar, $200 \mathrm{sccm}-\mathrm{H}_{2}, 10^{4} \mathrm{ppm}$. 
arbitrary constant, $C$, depends on instrumental function, electron energy distribution function (EEDF), and the electron-atom collision cross-section. As described in section 4, a detailed radiative-collision model is required for quantitative discussion. However, Eq. (3) is validated qualitatively and is used frequently for rough estimation of the number density of reactive species ${ }^{(15)}$. In this estimation, we maintained the hydrogen concentration so that EEDF and elementary inelastic processes remained unchanged.

The relative concentration of atomic silicon, $N_{S i}{ }^{\prime}$, is plotted with respect to initial $\mathrm{SiCl}_{4}$ concentration (Fig. 9). When the argon flow rate was $1000 \mathrm{~cm}^{3} \mathrm{~min}^{-1}, N_{S i}{ }^{\prime}$ was proportional to the initial $\mathrm{SiCl}_{4}$ content. The electron density $\left(10^{15} \mathrm{~cm}^{-3}\right)$ is 10 times greater than $\mathrm{SiCl}_{4}$ content. Therefore, $\mathrm{SiCl}_{4}$ is expected to decompose fully into atomic silicon. It is also evident that the as-synthesized material did not include chlorine $\left(<0.3\right.$ atomic\%) at $1000 \mathrm{~cm}^{3} \mathrm{~min}^{-1}$. On the other hand, $N_{S i}{ }^{\prime}$ dramatically decreased at $200 \mathrm{~cm}^{3} \mathrm{~min}^{-1}$ and was no longer a linear function of initial $\mathrm{SiCl}_{4}$ content, implying that atomic silicon is consumed via particle nucleation at lower flow rates. In other words, particle nucleation seemed to initiate in microplasma and the silicon nuclei continued growing, even as nitrogen and chlorine were incorporated as contamination as discussed in section 5.1. Although deposited particles are not mono-dispersed, individual silicon particles are visible in transmission electron micrographs (TEM). Silicon particle diameters were 3-15 nm (Figs. 10(a)-10(c)). Large particles like those shown in Fig. 10(c) exhibited silicon (111) whose surface energy is smallest among other orientations. Figure $10(\mathrm{~d})$ is a silicon polycrystal, suggesting that secondary nucleation on a silicon crystal is also possible. Individual particles are well separated and particle coagulation was not observed. Although silicon oxide was confirmed by the infrared spectra, the silicon particles shown in Fig. 10 are not encapsulated by an oxide layer.

\section{Concluding remarks}

A continuous-flow microplasma reactor using atmospheric pressure was designed for gas-phase synthesis of silicon nanocrystals. Optical emission spectroscopy showed that the electron density was as high as $10^{15} \mathrm{~cm}^{-3}$; the gas temperature was around $1500 \mathrm{~K}$. Such high-density non-thermal plasma decomposed silicon tetrachloride into atomic silicon within $6 \mu$ s and synthesized 3-15 nm silicon nanocrystals. Chlorine and nitrogen contaminated the as-synthesized material when the argon flow rate was $200 \mathrm{~cm}^{3} \mathrm{~min}^{-1}$. In this case, the product

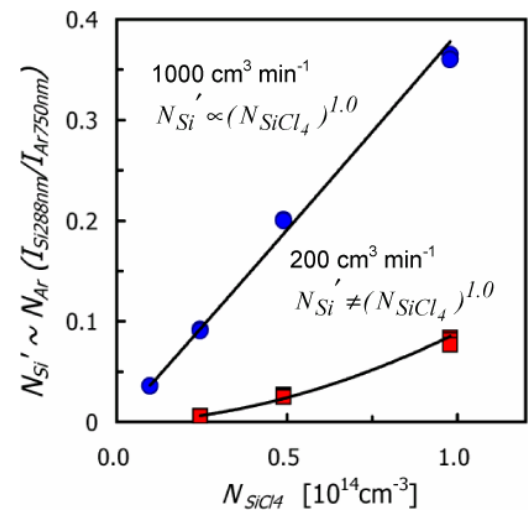

Fig. 9 Relative concentration of atomic silicon in a microplasma reactor estimated using actinometry: $\mathbf{n}, \operatorname{Ar} 200$ $\mathrm{cm}^{3} \min ^{-1}$; •, Ar $1000 \mathrm{~cm}^{3} \min ^{-1} ; \mathrm{H}_{2}$ was $10^{4} \mathrm{ppm}$.
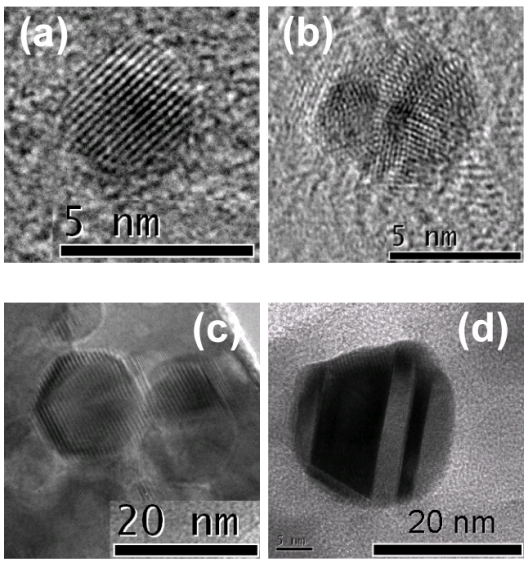

Fig. 10 High resolution TEM images of silicon nanocrystals with grain sizes of 3-15 nm. 
was mainly ammonium chloride $\left(\mathrm{NH}_{4} \mathrm{Cl}\right)$. Atomic concentration of nitrogen and chlorine was well correlated with the emission intensity of $\mathrm{NH}(336 \mathrm{~nm})$. The atomic concentration of silicon in the as-synthesized material increased to $75 \%$ with decreasing residence time (shorter than $10 \mu \mathrm{s}$ ): particle nucleation in the microplasma should be avoided to exclude nitrogen contamination. In addition, TEM analysis revealed individual 3-15 nm silicon nanocrystals. Although the silicon particles were not mono-dispersed, they were well separated and not coagulated.

\section{Acknowledgements}

This project was supported by Japanese Ministry of Education, Culture, Sports, Science and Technology Grants-in-Aid for Scientific Research on the Priority Area of Microplasmas (16040207) and for Young Scientists (A) (18686018). Particularly, T.N. would like to express thanks to Professor Hajime Shirai of Saitama University for intensive discussion of material characterization. Mr. Akira Genseki, of the Center for Advanced Materials Analysis of Tokyo Institute of Technology, supported TEM analysis.

\section{References}

[1] http://plasma.kuee.kyoto-u.ac.jp/tokutei429/

[2] Nozaki T., Sasaki K., Ogino T., Asahi D., Okazaki K., "Microplasma synthesis of tunable photoluminescent silicon nanocrystals", Nanotechnology, 18(23) (2007) 235603.

[3] Girshick S. L., Rao N. P., Kelkar M., "Model for ion-induced nucleation based on properties of small ionic clusters", J. Vac. Sci. Technol. A, 14(2) (1996), 529-534.

[4] Mangolini L., Thimsen E., Kortshagen U., "High Yield Plasma Synthesis of Luminescent Silicon Nanocrystals", Nano Letters, 5-4 (2005), 655-659.

[5] Xuegeng L., Yuanqing H., Talukdar S. S., Swihart M. T., "Process for Preparing Macroscopic Quantities of Brightly Photoluminescent Silicon Nanoparticles with Emission Spanning the Visible Spectrum", Langmuir, 19 (2003), 8490-8496.

[6] Makimura T., Mizuta T., Murakami K., "Laser Ablation Synthesis of Hydrogenated Silicon Nanoparticles with Green Photoluminescence in the Gas Phase", Jpn. J. Appl. Phys. 41 (2002), L144-L146.

[7] A. Von Engel, Ionized Gases (1955), Chapter 7, Oxford Press.

[8] Honda K., Gas discharge phenomena: 19th edition (1987), Chapter 4, Tokyo Denki University Press. (in Japanese)

[9] The Institute of Electrical Engineers of Japan, Handbook of discharge: 3rd edition (1980), 233-237, Ohmsha. (in Japanese)

[10] Yamamoto M., Murakami S., Plasma spectroscopy (1995), Japan Scientific Societies Press (in Japanese).

[11] Griem H. R., Plasma Spectroscopy (1964), Chapter 14, McGraw-Hill, New York.

[12] Nozaki T., Miyazaki Y., Unno Y., Okazaki K., "Energy Distribution and Heat Transfer Mechanisms in Atmospheric Pressure Non-equilibrium Plasmas", J. Phys. D: Appl. Phys., 34 (2001), 3383-3390.

[13] Nozaki T., Unno Y., Okazaki K., "Thermal Structure of Atmospheric Pressure Non-equilibrium Plasmas", Plasma Sources Sci. Technol., 11 (2002), 431-438.

[14] http://physics.nist.gov/PhysRefData/ASD/

[15] Coburn J. W., Chen M., "Optical emission spectroscopy of reactive plasmas: A method for correlating emission intensities to reactive particle density", J. Appl. Phys., 51 (1980), 3134-3136. 